\title{
Abordaje TAPP versus TEP en hernia inguinal unilateral. Experiencia de un Centro de Tercer Nivel
}

\author{
TAPP vs TEP in unilateral inguinal hernia. A single Tertiary Care Centre experience \\ Luis Angel Muciño Pérez, ${ }^{*}$ Carlos Alberto Santana Pérez, ${ }^{*}$ Lisa María Guzmán Alcántar, ${ }^{*}$ \\ Andrea Fernanda Ortega Juárez, ${ }^{*}$ Manuel Alejandro Pérez Ibáñez, ${ }^{*}$ César Jaramillo Martínez, ${ }^{\ddagger}$ Mario Betancourt Ángeles ${ }^{\ddagger}$ \\ * Centro Médico ISSEMyM de Toluca. \\ ₹ Servicio de Cirugía General, Centro Médico ISSEMyM de Toluca, Especialidad de Cirugía General UAEMéx.
}

RESUMEN

Introducción: La plastia inguinal es uno de los procedimientos más comunes en el mundo con un estimado de 20 millones de procedimientos realizados anualmente. Actualmente no existe un consenso común sobre cual técnica debe ser empleada sistemáticamente. Las técnicas laparoscópicas para la reparación de hernia inguinal ofrecen ventajas como menor dolor posoperatorio, menor tasa de complicaciones locales, mejor resultado estético, regreso anticipado a las actividades laborales. Material y métodos: Se realizó un estudio observacional retrospectivo con la programación quirúrgica de dos cirujanos con experiencia en plastia inguinal laparoendoscópica. Ambos equipos quirúrgicos realizaron los dos tipos de abordaje TAPP y TEP durante un periodo de enero de 2015 a diciembre de 2019. Se registraron datos preoperatorios en relación a edad, género, tipo de hernia y comorbilidades. De la cirugía se registró el tipo de técnica, el sangrado y la duración operatoria. Las complicaciones posoperatorias, el dolor posoperatorio durante la hospitalización y la duración de la misma se unificaron con los datos del seguimiento hasta los seis meses. Resultados: De un total de 100 pacientes con criterios de inclusión, 55 fueron sometidos a plastia tipo TAPP y 45 a abordaje TEP. Destaca de esta serie la tasa de complicaciones menores totales $8 \%$, infección de herida: $0 \%$; recidivas: $3 \%$. Neuralgia inguinal: $3 \%$. Conclusión: El uso de las técnicas laparoendoscópicas TAPP y TEP permite un abordaje seguro, eficaz y reproducible en nuestro medio para la reparación de hernias inguinales unilaterales con resultados comparables con los descritos en la literatura internacional.

Palabras clave: Plastia inguinal unilateral, laparoendoscópica, transabdominal preperitoneal, total extraperitoneal.
ABSTRACT

Introduction: Inguinal repair stands as one of the most common procedures worldwide with an estimate of 20 million procedures annually. Until today, there is no consensus about which technique should be used systematically. Laparoendoscopic techniques for inguinal repair offers advantages such as less postoperatory pain, less local complication rates, better cosmetic results and early comeback to work activities. Material and methods: Retrospective study with the surgical agenda from two experienced surgeons in the field of laparoendoscopic inguinal hernia repair. Both teams performed both TAPP and TEP approaches during the period of time between January 2015 and December 2019. Age, gender, hernia characteristics and comorbidities were documented. Technical aspects, blood loss and operatory time were taken from surgery reports. Postoperatory complications, pain during hospital stay, and duration of the latter, were taken into consideration with follow-up data up to six months. Results: From a total of 100 patients meeting the inclusion criteria, 55 underwent TAPP repair and 45 underwent TEP approach. From this series we get $8 \%$ of total minor complications, $0 \%$ with wound infection, $3 \%$ with relapse and $3 \%$ with inguinal neuralgia. Conclusion: Usage of TAPP and TEP laparoendoscopic techniques allows a safe, effective and replicable approach towards inguinal hernia repair with comparable results with those described in international literature.

Keywords: Unilateral inguinal repair, laparoendoscopic, transabdominal preperitoneal, total extraperitoneal.

\author{
Recibido para publicación: 13/08/2020. Aceptado: 07/10/2020. \\ Correspondencia: Dr. Luis Ángel Muciño Pérez \\ Av. Baja Velocidad Núm. 284, San Jerónimo Chicahualco, 52170, Metepec, México. \\ E-mail: mc.luismucino@gmail.com
}

Citar como: Muciño PLA, Santana PCA, Guzmán ALM, Ortega JAF, Pérez IMA, Jaramillo MC et al. Abordaje TAPP versus TEP en hernia inguinal unilateral. Experiencia de un Centro de Tercer Nivel. Rev Mex Cir Endoscop. 2020; 21 (1): 15-20. https://dx.doi.org/10.35366/97608. 


\section{INTRODUCCIÓN}

La plastia inguinal es uno de los procedimientos más comunes en el mundo con un estimado de 20 millones de procedimientos realizados anualmente. ${ }^{1,2}$ El riesgo vitalicio de presentar una hernia inguinal -la protrusión visceral o de tejido adiposo a través del canal inguinal o femoral- es de $27-43 \%$ en hombres y de $3-6 \%$ en mujeres. ${ }^{2}$ En varones mayores de 70 años, se estima una incidencia de 15:1,000. ${ }^{3}$

De acuerdo a la Sociedad Mexicana de la Hernia y el Sistema Nacional de Información en Salud, 5.85\% de la población en México presenta hernias inguinales, predominando en hombres entre 30-59 años (40-51\%) con una relación 2-3:1. La incarceración es la principal complicación con un riesgo de 7-30\%, y de éstas se estrangulan $10 \%$, aproximadamente. ${ }^{4}$

En general, todas las hernias inguinales son sintomáticas en menor o mayor grado y la única cura es la cirugía. ${ }^{2}$ De acuerdo con la Sociedad Europea de la Hernia, la vigilancia es una opción aceptable en el pequeño grupo de pacientes que se presentan asintomáticos o con síntomas leves, o en la presencia de comorbilidades mayores. Sin embargo $54-70 \%$ de los mismos requerirán manejo quirúrgico dentro de los primeros cinco años de vigilancia por empeoramiento de los síntomas, principalmente dolor. ${ }^{2,3}$ La conducta expectante también se acompaña de $2.5 \%$ de riesgo de incarceración acompañado de altas tasas de morbimortalidad. ${ }^{3}$

Actualmente no existe un consenso común sobre cuál técnica debe emplearse sistemáticamente. ${ }^{5}$ Existen más de 100 técnicas diferentes descritas para la plastia inguinal con diferentes indicaciones, clasificaciones y métodos. La recurrencia, el índice de complicaciones y la experiencia en una u otra técnica, condicionan al cirujano al momento de elegir una reparación. ${ }^{5}$ Las técnicas laparoscópicas para la reparación de hernia inguinal han sido reconocidas como una alternativa apropiada desde hace más de 20 años. ${ }^{6}$ Entre las ventajas que ofrecen: Menos dolor, menor requerimiento de analgésico, tasa más baja de infección de herida, mejor resultado estético, y regreso anticipado a las actividades laborales. Por el contrario, representan un gasto mayor en comparación con otras técnicas, además de requerir una curva de aprendizaje particular. ${ }^{5}$ Las guías más recientes del Hernia Surge Group únicamente recomiendan las técnicas laparoendoscópica de TEP y TAPP, el abordaje anterior de Lichtenstein y en casos seleccionados, la técnica de Shouldice. ${ }^{7}$

En nuestro centro, un hospital de referencia de tercer nivel, se ha implementado el uso de ambas técnicas de reparación laparoendoscópica. Describimos nuestra experiencia inicial a continuación.

\section{MATERIAL Y MÉTODOS}

Este estudio observacional retrospectivo se realizó en el Centro Médico ISSEMyM de Toluca en el Estado de México, con la programación quirúrgica de dos cirujanos con experiencia en plastia inguinal laparoendoscópica. Los dos equipos quirúrgicos realizaron ambos tipos de abordaje: transabdominal preperitoneal (TAPP) y total extraperitoneal (TEP) durante un periodo de enero de 2015 a diciembre de 2019.

Los criterios de inclusión del estudio fueron: 1) Edad: $>18$ años, ambos géneros, 2) Hernia: Directa e indirecta. Unilateral. Primarias y recidivas, 3) Cirugía: Electiva tipo TEP y TAPP. Los criterios de exclusión fueron: 1) Hernia: Femoral, 2) Cirugía: Otras técnicas de mínimo acceso como eTEP. Otros equipos quirúrgicos, 3) Pacientes con expedientes incompletos.

Se identificó un total de 113 pacientes operados en dicho periodo por plastia laparoendoscópica de hernia inguinal, de los cuales únicamente 100 cumplieron con los criterios de inclusión. No logró recabar datos suficientes del resto de los 13 pacientes excluidos (Tabla 1).

Se registraron datos preoperatorios en relación a la edad, el género, el protocolo diagnóstico, tipo de hernia y comorbilidades. De la cirugía se registró el tipo de técnica, el sangrado y duración operatoria.

Tabla 1: Características demográficas de la muestra.

Pacientes y características de las hernias

\begin{tabular}{lcc}
\hline & TAPP $(\mathrm{n})$ & TEP $(\mathrm{n})$ \\
\hline $\begin{array}{l}\text { Total de pacientes } \\
\text { Sexo }\end{array}$ & 55 & 45 \\
$\quad$ Masculino & 14 & 7 \\
$\quad$ Femenino & 41 & 38 \\
Edad (años) & & \\
<45 & 16 & 13 \\
46 a 60 & 23 & 15 \\
> 60 & 16 & 17 \\
Hernia & & \\
Indirecta & 49 & 33 \\
Directa & 6 & 12 \\
Localización & & \\
Derecha & & 22 \\
Izquierda & 27 & 23 \\
Tamaño (cm) & 28 & 8 \\
< 1 & & 24 \\
1 a 2 & 7 & 13 \\
> 3 & 27 & 42 \\
Primaria & 21 & 3 \\
Recurrente & 46 & 9 \\
\hline
\end{tabular}

TAPP = transabdominal preperitoneal, TEP = total extraperitoneal. 
Para el grupo TAPP se unificó el uso de lente de $30^{\circ}$, pinzas de disección y retracción laparoscópica y equipo de laparoscopía convencionales, malla de polipropileno preformada en la mayoría de los casos, sistema de fijación con grapas helicoidales así como sutura barbada 2-0 para el cierre de flap peritoneal. Para el grupo TEP se utilizó el mismo material, con excepción de la sutura y agregando el dispositivo de acceso y disección con globo. Por elección de los cirujanos, se realizó fijación de la malla con grapas helicoidales en este último abordaje, excepto en los casos selectos en los que se utilizaron mallas autoadheribles.

Las complicaciones posoperatorias, el dolor posoperatorio durante la hospitalización y la duración de la misma se unificaron con los datos del seguimiento hasta seis meses.

Análisis estadístico. Se realizó con la herramienta SPSS IBM versión 20.0. Los datos se presentan como o media \pm DE o porcentaje. El resto de las variables se asociaron mediante un análisis de varianza de una vía, y las complicaciones se expresan en porcentaje de la muestra total.

\section{RESULTADOS}

Entre enero de 2015 y diciembre de 2019, un total de 100 pacientes se sometió a plastia inguinal con técnicas laparoendoscópicas con criterios de inclusión para nuestro estudio, de los cuales 55 casos fueron sometidos a plastia inguinal tipo TAPP y 45 a abordaje TEP.

La edad media del grupo TAPP fue de $51.5 \pm 14.9$ años, mientras que en el grupo TEP fue de $55.6 \pm 16.1$ años. No existe diferencia estadística significativa entre los dos grupos con respecto a la edad ( $p=0.19)$. En ambos grupos predominaron los varones con 76.3 y $84.4 \%$ en el grupo TAPP y TEP, respectivamente.

Del grupo TAPP, 49 pacientes presentaban hernias indirectas frente a 33 del grupo TEP. Únicamente $16.3 \%$ del grupo TAPP se operaba por recidiva de hernia inguinal, todos con antecedente de abordaje anterior; mientras que en el grupo TEP, $6.6 \%$ presentaba esta situación.

En el grupo TAPP la media de tiempo operatorio fue de $31.6 \pm 3.7$ minutos, mientras que en el grupo TEP fue de 28.8 \pm 3.8 minutos con una relevancia estadística $p=0.00047$. Sangrado reportado alrededor de $10 \mathrm{~cm}^{3}$ en toda la población. No se utilizó ningún tipo de drenaje en ningún procedimiento.

Por políticas institucionales, la dieta enteral se reinicia hasta el ingreso a piso con un aproximado de seis horas posterior al acto quirúrgico. La media de la hospitalización fue de $1.05 \pm 0.2$ días para el grupo TAPP, mientras que para el grupo TEP se elevó hasta $1.3 \pm 0.9$ días, sin significancia estadística $(p=0.06)$.

La medición de la escala visual análoga del dolor para el grupo TAPP reportó media de $4.7 \pm 0.6$ puntos, mientras que en el grupo TEP se documentó una media de $4.9 \pm$
Tabla 2: Resumen de resultados de ambas técnicas.

\begin{tabular}{lcc} 
& TAPP & TEP \\
\hline Número de pacientes & 55 & 45 \\
Edad media (años) & $51.5 \pm 14.9$ & $55.6 \pm 16.1$ \\
Género (\%) & 76.3 & 84.4 \\
$\quad$ Masculino & 23.7 & 15.6 \\
$\quad$ Femenino & 12.7 & 6.6 \\
Abordaje anterior previo (\%) & 31.6 & 28.8 \\
Tiempo operatorio (min) & 10 & 10 \\
Sangrado (mL) & $1.05 \pm 0.2$ & $1.3 \pm 0.9$ \\
Días hospitalización (media) & & \\
EVA, horas (puntos) & $4.7 \pm 0.6$ & $4.9 \pm 0.7$ \\
0-12 & $3.5 \pm 0.9$ & $3.7 \pm 0.8$ \\
12-24 & 3.6 & 2.2 \\
Hematomas (\%) & 7.2 & 6.6 \\
Seromas (\%) & 3.6 & 2.2 \\
Recidivas (\%) & 3.6 & 2.2 \\
Dolor crónico (\%) & & \\
\hline
\end{tabular}

TAPP $=$ transabdominal preperitoneal, TEP = total extraperitoneal.

0.7 puntos, entre las 0 y 12 horas del posoperatorio. Para el periodo entre las 12 y 24 horas, la media para el grupo TAPP fue $3.5 \pm 0.9$ puntos y para el grupo TEP de $3.7 \pm 0.8$ puntos. Todas las mediciones se realizaron bajo analgesia convencional con acetaminofén, AINE y hielo local.

En el grupo TAPP se registraron complicaciones en la siguiente proporción: Hematomas 3.6\% (dos pacientes); seromas $7.2 \%$ (cuatro casos); no se registraron infecciones de la herida quirúrgica. En el grupo TEP se registraron seromas en $6.6 \%$ (tres pacientes), hematomas en $2.2 \%$ (un caso) y tampoco se registraron infecciones de la herida.

Finalmente, se documentaron $3.6 \%$ de recidivas en el grupo TAPP por $2.2 \%$ del grupo TEP. El $3.5 \%$ de los pacientes presentó dolor crónico (más de tres meses) en el grupo TAPP y $2.2 \%$ en el grupo TEP durante el seguimiento. El resumen de los resultados se muestra en la Tabla 2.

\section{DISCUSIÓN}

Basado en las más recientes guías internacionales para el manejo de la hernia inguinal no existe un consenso para indicar una única técnica que sea adecuada para las características de todos los pacientes. Es por eso que se deben idear tratamientos personalizados con base en: sexo del paciente, localización y tipo de hernia, antecedente de procedimientos abdominales y pélvicos, comorbilidades cardiopulmonares mayores y recurrencia.

Las técnicas laparoscópicas han demostrado ser superiores a las técnicas abiertas, disminuyen el dolor posoperatorio, inguinodinia a largo plazo, tiempo de hospitalización más corto, menor índice de recurrencias, menor formación 
de seromas, hematomas, e infección de la herida quirúrgica, resultando en una pronta recuperación con incorporación temprana a las actividades diarias; seguimiento en cinco años de la técnica Lichtenstein vs. TEP, durante el que se observó dolor crónico de 18.8 vs. 9.4\%; y Lichtenstein vs. TAPP con seguimiento a 53 meses, con dolor leve de 23.7 vs. $14.8 \%$, dolor moderado de 5.3 vs. $1.2 \%$, dolor severo de 3.9 vs. $0 \%{ }^{8}$ Es por ello que al encontrarnos en un centro de referencia de tercer nivel, es de vital importancia incorporar dentro de lo posible dichas ventajas a los procedimientos que ofrecemos a la población.

Se han descrito dos variables inherentes al paciente que se corresponden con el éxito de las plastias laparoendoscópicas; en primer lugar, condiciones como obesidad, sexo femenino, edad mayor de 80 años, presencia de comorbilidades, las cuales confieren una puntación ASA elevada; y segundo, el tipo de hernia como reparaciones de emergencia, hernias bilaterales, defecto largo o antecedente de cirugía abdominal baja previa, los cuales agregan cierto grado de complejidad, ${ }^{7}$ y se relacionan con tiempo quirúrgico más prolongado y mayor número de complicaciones severas con respecto a la afección visceral. ${ }^{9}$ Se considera que la curva de aprendizaje estimada para la técnica TEP se estabiliza después de 40 a 100 procedimientos; mientras que los tiempos de operación del procedimiento TAPP se estabilizan después de 30 a 50 procedimientos, ${ }^{10}$ por lo que la implementación reciente en un centro hospitalario como el nuestro se debe hacer con la mayor atención a la selección rigurosa de pacientes con factores favorables para un procedimiento exitoso.

Existen dos técnicas estándar para la reparación laparoscópica de hernia inguinal: La reparación preperitoneal transabdominal (Figura 1) y la técnica total extraperitoneal (Figura 2). ${ }^{6}$ Otras modificaciones como la eTEP con abordaje extendido, la SILS-TEP con una incisión única y versiones robóticas ${ }^{11}$ exceden el propósito de nuestro estudio.

El equipo estándar requerido para plastia inguinal laparoscópica consta de una sonda urinaria para descomprimir la vejiga, lo que permite una adecuada exposición y seguridad, aguja de Veress o trocar de Hasson de 12 mm, dependiendo del abordaje inicial preferido, trocares accesorios de $5 \mathrm{~mm}\left(\chi^{2}\right)$, pinzas de agarre, disector Maryland, tijeras Metzenbaum, gancho de electrocauterio, portaaguja, laparoscopio de $30^{\circ}$, un sistema de irrigación y succión. ${ }^{12}$ Malla del material y preferencia del cirujano, siendo más utilizada por nosotros la malla de monofilamento de polipropileno preformada y precortada, clips hemostáticos, un dispositivo de fijación, siendo éste con pegamento o con tackers; el cierre del flap con sutura barbada es conveniente porque evita tensionar, desgarrar y disminuir el tiempo requerido al momento del cierre. Para TEP existen diferentes sistemas
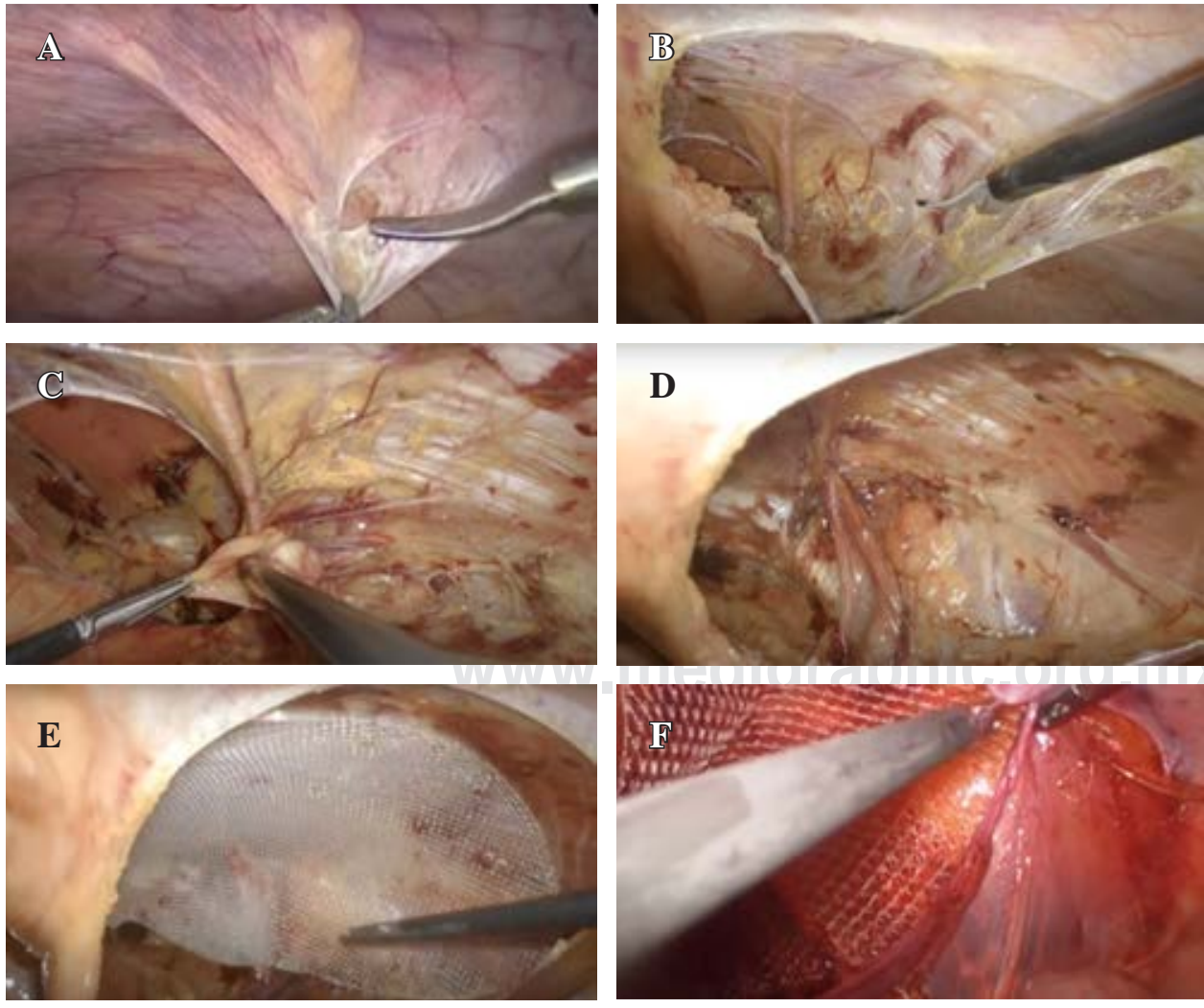

Figura 1:

Técnica TAPP. A y B) Apertura del flap peritoneal y disección del espacio preperitoneal con ayuda del neumoperitoneo. C) Disección del saco herniario de las estructuras del cordón espermático. D y E) Disección completa del orificio miopectinio para colocación de malla. F) Cierre del flap peritoneal.

TAPP = transabdominal preperitoneal 

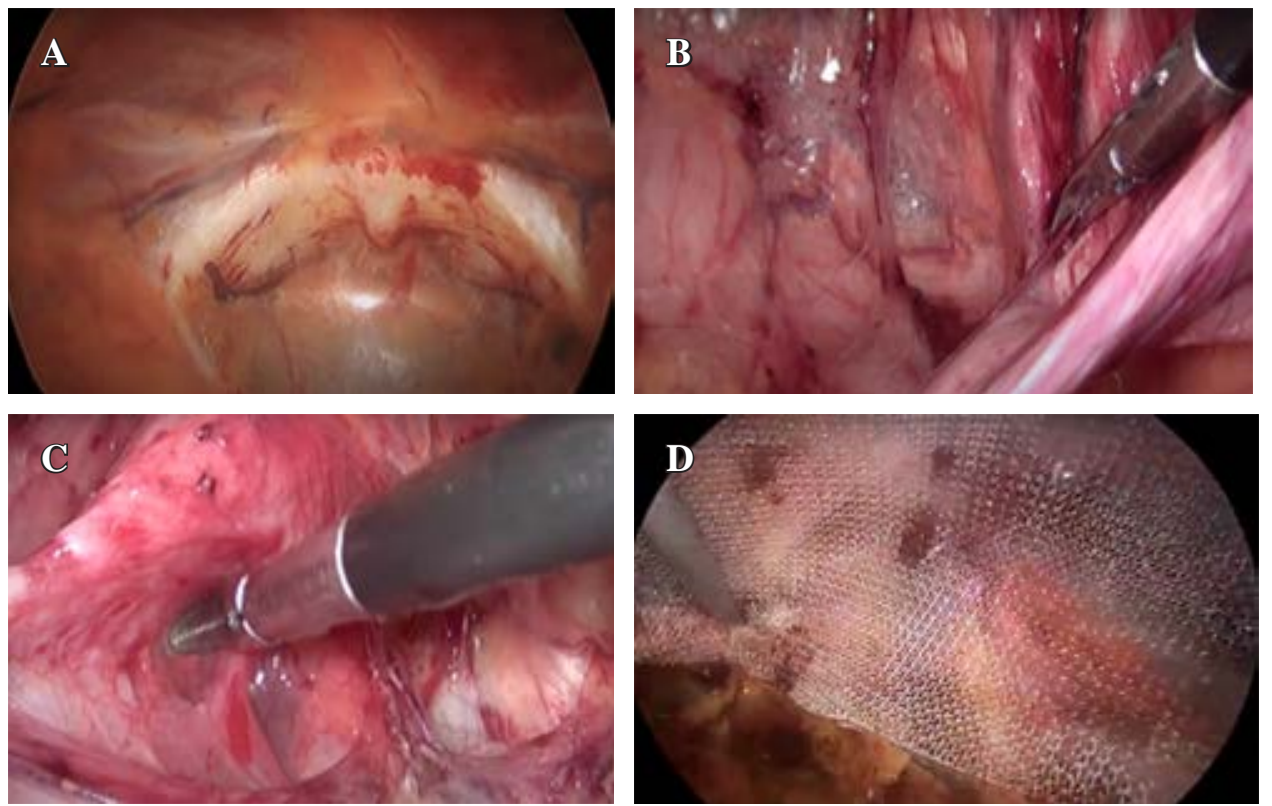

Figura 2:

Técnica TEP. A) Disección del espacio preperitoneal con globo disector/ hemostático. B) Disección del saco herniario de las estructuras del cordón espermático. C) Disección de espacio para la colocación de malla cubriendo la totalidad del orificio miopectíneo. D) Colocación y fijación de malla. $\mathrm{TEP}=$ total extraperitoneal.

para la disección preperitoneal, entre los más completos se encuentran los sistemas de acceso y disección con globo. ${ }^{13}$

En la técnica TAAP, introducida por Arregui y Dion en 1990, es necesario acceder a la cavidad abdominal creando neumoperitoneo; después se incide el peritoneo liberando un flap del mismo, permitiendo la disección neumática del espacio, se diseca el saco herniario y se coloca una malla en el espacio preperitoneal que cubra el orificio miopectineo en su totalidad. Finalmente, el peritoneo se cierra cubriendo la malla. ${ }^{1}$ Con la técnica TAPP, se han reportado más complicaciones como lesiones viscerales, tiempo operatorio más prolongado, mayor incidencia de edema de cordón y formación de seromas y mayor dolor posoperatorio. ${ }^{6,14} \mathrm{El}$ tiempo descrito en la literatura va de 46.3 a 73.7 minutos en distintas series desde 1998 hasta 2012. ${ }^{15}$ Para el dolor posoperatorio inmediato se han reportado medias de 3.8 a 4.4 en la escala visual análoga a las $0-12$ horas y de 1.4 a 2.3 en diversas series. ${ }^{15}$ Aproximadamente $6.1 \%$ reporta formación de hematomas, $6.2 \%$ de seromas y $0.94 \%$ infecciones de herida quirúrgica. ${ }^{15}$ Diversos estudios reportan una estancia hospitalaria de 3.5 a cinco días, ${ }^{15}$ en parte cesgado por las diversas políticas hospitalarias como se comenta en dichos reportes. Finalmente, se ha documentado una recurrencia alrededor de $2.6 \% .^{15}$ Los resultados tomados de nuestro estudio se mantienen dentro del rango de una desviación estándar con base en los reportados de la literatura internacional.

La técnica laparoendoscópica total extraperitoneal, realizada por primera vez por Duluq en 1992, permite la visualización del espacio miopectineo, la disección, reducción del saco herniario y su contenido, sumado a la colocación de una malla, con el beneficio teórico de evitar entrar a la cavidad abdominal; ${ }^{14}$ combina las ventajas de una reparación libre de tensión al emplear una malla en la región inguinal con las ventajas de la cirugía de mínimo acceso con menor dolor posoperatorio y un periodo de recuperación más corto, ${ }^{11,16}$ pero con el inconveniente de requerir una curva de aprendizaje específica e insumos adicionales para su realización. ${ }^{6}$ La identificación topográfica de la anatomía es clave para su realización; se deben identificar los vasos hipogástricos, el pubis, los elementos del cordón espermático. Posteriormente se crea un espacio entre los elementos del cordón y por encima del iliopsoas. Por último, se reduce el saco y se coloca una malla cubriendo el defecto en el espacio previamente creado. Se puede realizar fijación de la misma hacia el ligamento de Cooper, el tubérculo del pubis, el área conjunta y los músculos rectos del abdomen, ${ }^{11}$ con sus respectivas modificaciones con base en las guías recientes. Si bien el abordaje representa menor riesgo de lesión visceral, existe mayor riesgo de sangrado durante la disección del espacio preperitoneal que además puede condicionar la visión durante el procedimiento. Para esta técnica, en 2018, en un metaanálisis, Gavriilidis y colaboradores describieron un tiempo quirúrgico aproximado de 30-48 minutos, 2.4$6 \%$ de recurrencia, $11 \%$ de dolor crónico, formación de hematoma en $2.9-12 \%$ y seroma en $4.6-5 \%$, infección de herida quirúrgica en 1-2\%, lesión vascular 0.5-1.3\% y dolor persistente en $11-13 \%{ }^{17}$

Existe una tendencia creciente a realizar dicho abordaje con el paciente bajo anestesia regional o espinal, con resul- 
tados prometedores, principalmente bajo dos directrices: 1) Extender el criterio de cirugía de mínimo acceso a casos con comorbilidades mayores o riesgo anestésico elevado. 2) Considerar realizar estos procedimientos de manera ambulatoria, disminuyendo los costos de hospitalización. ${ }^{5}$ Esta situación presenta un campo de investigación incipiente para nuestra población hospitalaria en un futuro.

La recidiva de hernias en México va de 11-20\% con técnicas de tensión y de 0.5 -5\% con técnicas sin tensión abiertas o laparoscópicas. ${ }^{4}$ En nuestro centro registramos una recidiva global de 3\% para las técnicas laparoendoscópicas, entrando en el rango descrito para la literatura nacional.

Existen múltiples estudios que comparan la seguridad y eficacia de una y otra técnicas; sin embargo, encontramos diversos resultados en ambas, no existiendo un consenso sobre cuál técnica puede emplearse de manera sistemática. ${ }^{6}$ Las complicaciones reportadas en nuestro estudio no muestran diferencias relevantes entre ambas técnicas. Durante la recolección de datos, la narrativa de los mismos documenta que la mayor incidencia de complicaciones como seromas y hematomas se dio en mayor parte en los primeros casos, durante el periodo de adecuación de la técnica, siendo éstos prácticamente nulos en los últimos dos años de registro. Asimismo, los casos con sacos herniarios amplios, la disección cruenta, los pacientes obesos y las recidivas ${ }^{7}$ coinciden con la presentación de dichas complicaciones, condiciones propiamente descritas en la literatura.

\section{CONCLUSIONES}

El uso de las técnicas laparoendoscópicas TAPP y TEP permite un abordaje seguro, eficaz y reproducible en nuestro medio para la reparación de hernias inguinales. Ambas técnicas requieren de curva de aprendizaje específica, insumos adicionales y representan riesgos particulares asociados. La experiencia en nuestro centro es equivalente a la literatura internacional, con resultados funcionales y tasas de complicaciones comparables entre las técnicas TEP y TAPP para nuestra población.

\section{REFERENCIAS}

1. Tulin A, Slavu I, Braga V, Mihaila D, Alecu L. TAAP vs. TEP in inguinal hernia repair-what is the evidence? A single center experience. Chirurgia (Bucur). 2019; 114: 67-72. Available in: https://doi.org/10.21614/chirurgia.114.1.67.

2. HerniaSurge Group. International guidelines for groin hernia management. Hernia: the journal of hernias and abdominal wall surgery. Hernia. 2018; 22: 1-165. Available in: https://doi.org/10.1007/s¹0029-017-1668-x.

3. Voorbrood CE, Burgmans JP, Clevers GJ, Davids PH, Verleisdonk EJ, van Dalen T. Totally extraperitoneal (TEP) endoscopic hernia repair in elderly patients. Hernia. 2015; 19: 887-891. doi: 10.1007/s¹0029-015-1422-1.
4. Guías de práctica clínica para hernias de la pared abdominal. Asociación Mexicana de Hernia, A.C. Disponible en: http://amhernia.org/wp-content/themes/amhernia/ files/ guias2015.pdf.

5. Yildirim D, Hut A, Uzman S et al. Spinal anesthesia is safe in laparoscopic total extraperitoneal inguinal hernia repair. A retrospective clinical trial. Wideochir Inne Tech Maloinwazyjne. 2017; 12: 417-427. Available in: doi:10.5114/wiitm.2017.72325.

6. Krishna A, Bansal VK, Misra MC, Prajapati O, Kumar S. Totally extraperitoneal repair in inguinal hernia: more than a decade's experience at a tertiary care hospital. Surg Laparosc Endosc Percutan Tech. 2019; 29: 247-251. doi: 10.1097/SLE.0000000000000682.

7. Köckerling F. TEP for elective primary unilateral inguinal hernia repair in men: what do we know? Hernia. 2019; 23 : 439-459. doi: 10.1007/s 0029-019-01936-6.

8. Simons MP, Smietanski M, Bonjer HJ et al. International guidelines for groin hernia management. Hernia. 2018; 22: 1-165. Available in: https://doi.org/10.1007/sº029-0171668-x.

9. McCormack K, Wake B, Perez J et al. Laparoscopic surgery for inguinal hernia repair: systematic review of effectiveness and economic evaluation. Health Technol Assess. 2005; 9: 3-4. doi:10.3310/hta9140.

10. Burcharth J. The epidemiology and risk factors for recurrence after inguinal hernia surgery. Dan Med J. 2014; 61: B4846.

11. Łomnicki J, Leszko A, Kuliś D, Szura M. Current treatment of the inguinal hernia-the role of the totally extraperitoneal (TEP) hernia repair. Folia Med Cracov. 2018; 58: 103-114. Available in: https://doi.org/10.24425/fmc.2018.125076.

12. Jones DB. Master techniques in surgery: hernia. Philadelphia: Lippincott Williams \& Wilkins; 2012.

13. Sherwinter DA. Laparoscopic inguinal hernia repair. Medscape. 2020. Available in: https://emedicine.medscape. com/article/1534321-periprocedure\#b1.

14. Vărcuş F, Duţă C, Dobrescu A, Lazăr F, Papurica M, Tarta C. Laparoscopic repair of inguinal hernia TEP versus TAPP. Chirurgia (Bucur). 2016; 111: 308-312.

15. Scheuermann U, Niebisch S, Lyros O, Jansen-Winkeln B, Gockel I. Transabdominal preperitoneal (TAPP) versus Lichtenstein operation for primary inguinal hernia repair - A systematic review and meta-analysis of randomized controlled trials. BMC Surg. 2017; 17: 55. Available in: https://doi.org/10.1186/s'2893-017-0253-7.

16. Meyer A, Bonnet L, Bourbon M, Blanc P. Totally extraperitoneal (TEP) endoscopic inguinal hernia repair with TAP (transversus abdominis plane) block as a day-case: a prospective cohort study. Journal of Visceral Surgery. 2015; 152: 155-159. Available in: https://doi.org/10.1016/j. jviscsurg.2014.12.005.

17. Gavriilidis P, Davies RJ, Wheeler J, de'Angelis N, Di Saverio S. Total extraperitoneal endoscopic hernioplasty (TEP) versus Lichtenstein hernioplasty: a systematic review by updated traditional and cumulative meta-analysis of randomisedcontrolled trials. Hernia. 2019; 23: 1093-1103. Available in: https://doi.org/10.1007/s0029-019-02049-w. 